\title{
Colorectal anastomotic perforation secondary to acute ruptured appendicitis presenting as septic arthritis
}

\author{
Hryhoriy Bohdanovych Zhoba*1, Brian P. Fleischer ${ }^{2}$, Wesley B. Vanderlan ${ }^{3,4}$ \\ ${ }^{1}$ Florida State University, United States \\ ${ }^{2}$ Tulane University, United States \\ ${ }^{3}$ Uniformed Services University of the Health Sciences, United States \\ ${ }^{4}$ Louisiana State University, New Orleans, United States
}

Received: April 3, 2019

DOI: $10.5430 /$ css.v5n2p1
Accepted: June 12, 2019

Online Published: July 2, 2019

URL: https://doi.org/10.5430/css.v5n2p1

\begin{abstract}
Anastomotic leaks following abdominoperineal resection with rectal anastomosis become clinically significant in $2.9 \%-22 \%$ of cases. Local recurrence of cancer and local inflammation are the most common causes of these leaks . Colonic perforation presenting with suppurative involvement of the lower extremities has been previously reported. We describe herein the case of a colorectal anastomotic leak secondary to pathology-proved acute appendicitis presenting with suppurative necessitation causing right hip septic arthritis five years following lower anterior resection (LAR) for stage unspecified colorectal cancer. No similar case has been demonstrated in the surveyed literature.
\end{abstract}

Key Words: Colorectal anastomosis, Acute appendicitis, Septic arthritis, Lower anterior resection

\section{INTRODUCTION}

Anastomotic leaks after colorectal resection become clinically significant in $2.9 \%-22 \%$ of cases $^{[1,2]}$ with local recurrence of cancer and local inflammation serving as the most common causes of these leaks. ${ }^{[3]}$ Anastomotic complications may occur both in the immediate and remote post-operative period, requiring adequate and timely surveillance. Intestinal perforation may present involving lower extremity complaints. ${ }^{[4-7]}$ We present the case of a 66-year-old male with septic arthritis of the right hip secondary to pathology-proved acute ruptured appendicitis causing colorectal anastomotic perforation and subsequent suppurative fecal necessitation through the pelvis and right lower extremity occurring five years following lower anterior resection (LAR) with adjuvant chemoradiation therapy for stage unspecified colorectal cancer.

\section{CASE REPORT}

The 66-year-old patient presented to his local emergency department (ED) with a two-week history of idiopathic right hip pain. Pain progressed until he was unable to bear weight on the affected limb. The patient was diagnosed with septic arthritis of the right hip and referred to our institution. Past medical history was notable for stage unspecified colorectal treated with LAR five years prior to presentation with adjuvant chemoradiation.

On arrival at our institution, the patient endorsed worsening right hip pain over the previous two weeks. He specifically denied fever, abdominal and back pain, nausea and vomiting. Physical exam was notable for erythema, warmth, and induration overlying the right greater trochanter and lateral thigh. No right hip decreased range of motion was appreci-

*Correspondence: Hryhoriy Bohdanovych Zhoba; Email: greg.zhoba@ hotmail.com; Address: Florida State University, United States. 
ated. Abdominal exam was remarkably benign. Laboratory findings revealed an elevated white blood cell (WBC) count of 22,700. Erythrocyte sedimentation rate was elevated at 52 $\mathrm{mm} / \mathrm{hr}$. Roentgenograms revealed pneumoperitoneum and subcutaneous emphysema of the right thigh and hemipelvis. CT imaging of the abdomen and pelvis with inclusion of the proximal lower extremities confirmed colorectal perforation with fluctuant necessitating through the gluteal musculature (see Figure 1) and extending into the deep structures of the pelvis and right lower extremity (see Figures $2 \& 3$ ). The patient was taken emergently for exploratory laparotomy with attendant incision and debridement of deep right thigh abscess.

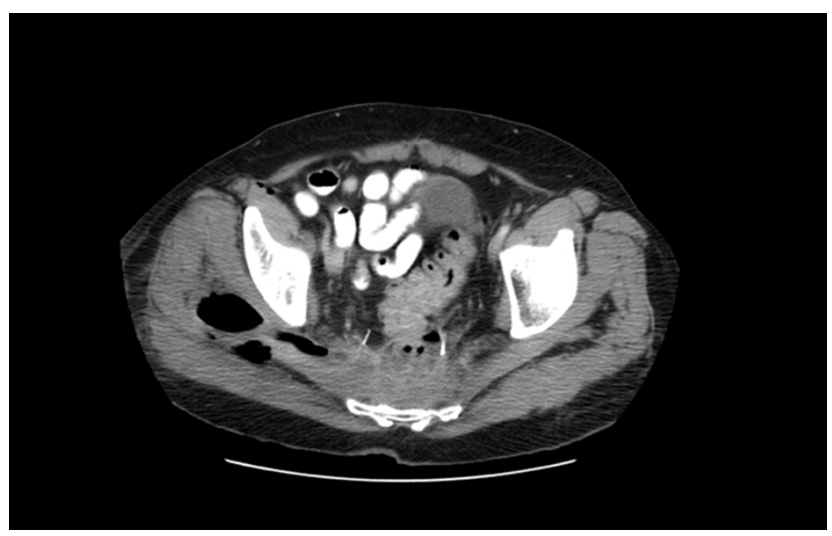

Figure 1. Axial cut of pre-operative abdominal and pelvic CT scan demonstrating a fistula emanating from the large bowel and tracking to the gluteal musculature

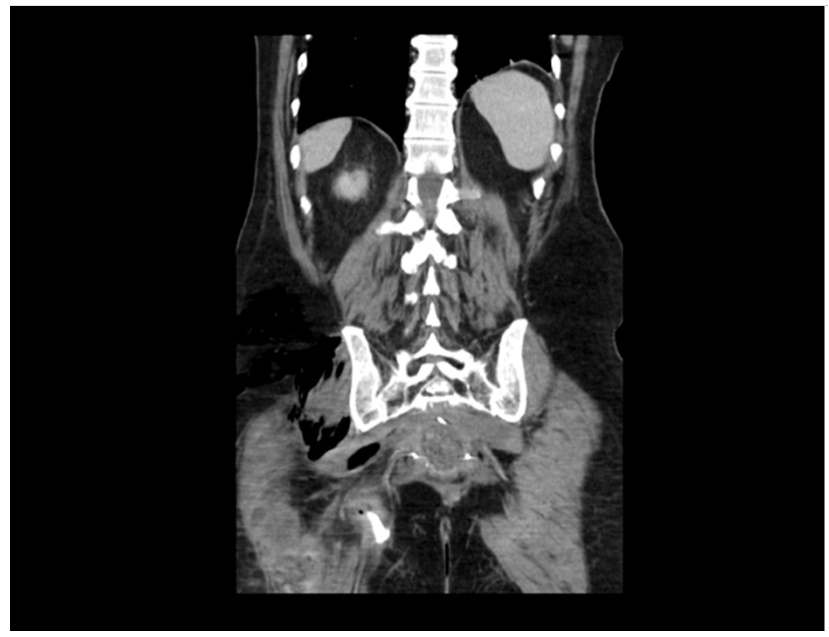

Figure 2. Sagittal cut of pre-operative abdominal and pelvic CT scan demonstrating involvement of the deep structures of the pelvis and thigh with the anastomotic leak

Midline laparotomy incision was performed using Bovie electrosurgery in standard fashion. Upon entry into the abdominal cavity, the small bowel was inspected in its entirety and was found free of intrinsic disease. The remaining viscera were examined and also found without evidence of intrinsic disease save the colon. The inflamed appendix was adhesed to the prior colorectal anastomosis. Dense fibrosis and adhesions between the appendix and the colorectal anastomosis indicated an underlying chronic inflammatory process. Rupture of the appendix tip was grossly obvious and contiguous with right anastomotic disruption. Fistulous tracking of suppuration and stool through the perineum and into the patient's right pelvis and proximal right lower extremity was also grossly obvious. Damage control proceedings were elected. Source control was achieved via sigmoid colectomy. Abdominal and perineal packing was placed and the anus was over sewn. There was no evidence of diverticular disease. Temporary abdominal closure was afforded using the negative pressure wound therapy ABTHERA ${ }^{T M}$ (KCI, Inc., San Antonio, TX) system. Orthopedic Surgery then presented to the operating room for debridement of the deep tissues of the right hip. Significant fat necrosis was identified superficial to the Iliotibial (IT) band. Copious fecal purulence poured from the wound upon IT incision and continued with blunt dissection of the quadriceps femoris and external hip rotators. All necrotic tissue was sharply debrided. The wound was copiously irrigated with normal saline and a vacuum-assisted wound closure (VAC) device (KCI, San Antonio, TX) was placed using GRANUFOAM ${ }^{T M}$ (KCI, San Antonio, TX). The patient tolerated both portions of this procedure without complications.

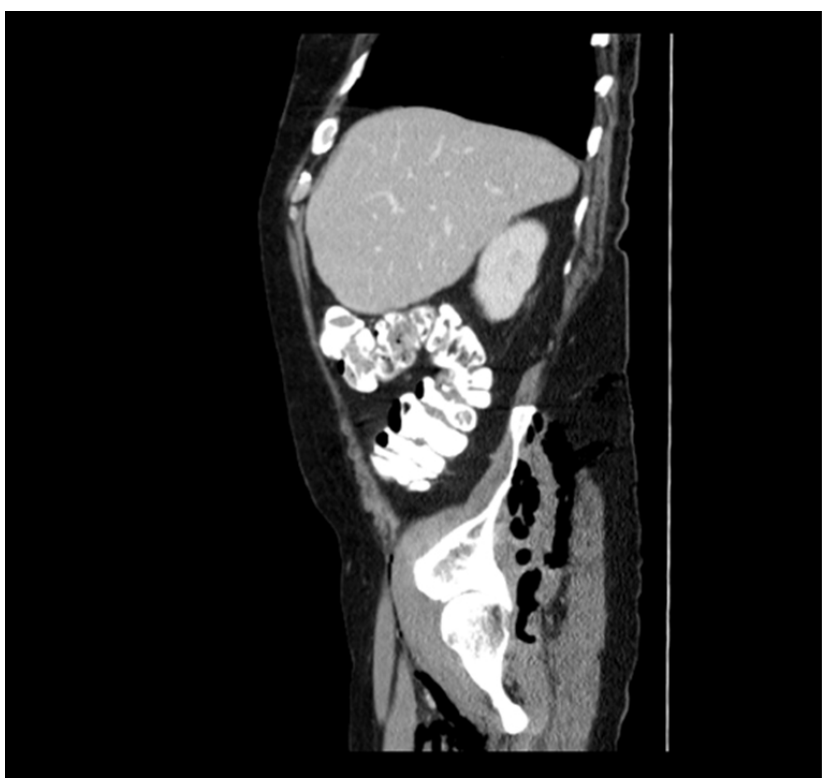

Figure 3. Coronal section or pre-operative abdominal and pelvic CT scan demonstrating anastomotic leak with a perianastomotic fistula necessitating feces into his right pelvis and thigh musculature 
On the fifth postoperative day, the patient returned to the operating room for the maturation of an end colostomy and completion perineal resection. The fascia was closed and a wound VAC with GRANUFOAM ${ }^{T M}$ was placed in the open wound. The patient tolerated these procedures well and without complications. Coordinated wound VAC changes with the orthopedic surgery service occurred regularly. The pathology returned a diagnosis of acute ruptured appendicitis with colorectal anastomotic perforation and no evidence of malignancy or diverticular disease. Recovery was uncomplicated and the patient was transferred to rehabilitation with subsequent loss to follow-up.

\section{DiscuSSION}

Anastomotic leaks become clinically significant in $2.9 \%$ $22 \%$ of cases $^{[1,2]}$ involving colorectal anastomoses. Local recurrence of cancer and local inflammation are the most commonly causal agents. ${ }^{[3]}$ Colorectal anastomotic perfora- tion secondary to ruptured acute appendicitis has not been previously reported. Colorectal anastomotic leaks most often present between the fifth and eighth post-operative days. ${ }^{[2]}$ The patient in this case report presented five years following LAR for stage unspecified colorectal cancer with adjuvant chemoradiation. Examination intraoperatively suggested that the appendix had become chronically adhesed to the colorectal anastomosis with subsequent development of acute appendicitis with suppurative rupture through the anastomosis. Intestinal perforation diagnosed with presenting complaints of subcutaneous emphysema, ${ }^{[4,5]}$ cellulitis, ${ }^{[6]}$ and enterocutaneous fistula ${ }^{[7]}$ have been reported. This case of a ruptured colorectal anastomosis secondary to acute ruptured appendicitis was uniquely diagnosed as right hip septic arthritis, thereby expanding the differential diagnosis of the presenting complaint.

\section{Conflicts of InTEREST Disclosure}

The authors declare they have no conflicts of interest.

\section{REFERENCES}

[1] Chambers WM, Mortensen NJ. Postoperative Leakage and Abscess Formation after Colorectal Surgery. Best Pract Res Clin Gastroenterol. 2004 Oct; 18(5): 865-80. https ://doi.org/10.1016/S1 521-6918(04)00087-3

[2] Daams F, Luyer M, Lange JF. Colorectal anastomotic leakage: Aspects of prevention, detection and treatment. World J Gastroenterol. 2013 Apr 21; 19(15): 2293-2297. PMid: 23613621. https : //doi.org/10.3748/wjg.v19.i15.2293

[3] Miccini M, Borghese O, Scarpini M, et al. Anastomotic leakage and septic complications: impact on local recurrence in surgery of low rectal cancer. Ann Ital Chir. 2011 Mar-Apr; 82(2): 117-23.

[4] Saldua NS, Fellars TA, Covey DC. Case Report: Bowel Perforation Presenting as Subcutaneous Emphysema of the Thigh. Clin
Orthop Relat Res. 2010 Feb; 468(2): 619-623. PMid: 19653051 https : //doi.org/10.1007/s11999-009-1015-3

[5] Jones GH, Kalaher HR. Diverticular Disease Presenting as Subcutaneous Emphysema of the Thigh. BMJ Case Rep. 2009; 2009: bcr01.2009.1465. PMid: 21687014. https://doi.org/10.1136/ bcr.01.2009.1465

[6] Karampinas PK, Evangelopoulos DS, Benetos IS, et al. RectoFemoral Fistula Presenting as Emphysematous Cellulitis of the Knee: A Case Report \& Literature Review. Case Rep Emerg Med. 2011; 2011: 479209. PMid: 23326693. https://doi .org/10.1155/20 $11 / 479209$

[7] Mayfield LH, Waugh JM. Sigmoidocutaneous Fistulae Resulting from Diverticulitis of the Sigmoid Colon. Ann Surg. 1949 Feb; 129(2): 198-206. PMid: 17859299. https://doi.org/10.109 7/00000658-194902000-00004 